\title{
Docking in Metal-Organic Frameworks
}

\author{
Qiaowei Li ${ }^{1 *}$ Wenyu Zhang, ${ }^{1 *}$ Ognjen Š. Miljanić, ${ }^{1}$ Chi-Hau Sue, ${ }^{2}$ Yan-Li Zhao, ${ }^{2}$ Lihua Liu, ${ }^{2}$ \\ Carolyn B. Knobler, ${ }^{1}$ J. Fraser Stoddart, ${ }^{2}$ Omar M. Yaghi ${ }^{1}$ \\ ${ }^{1}$ Department of Chemistry and Biochemistry, University of California-Los Angeles, Los Angeles, CA 90095, USA. \\ ${ }^{2}$ Department of Chemistry, Northwestern University, Evanston, IL 60208, USA.
}

*To whom correspondence should be addressed. Email: qwli@chem.ucla.edu (Q.L.); wyzhang@ @ chem.ucla.edu (W.Z.)

Metal-organic frameworks containing electron-donating macrocyclic polyethers are capable of docking incoming electron-accepting substrates in a stereoelectronically controlled fashion. 
The use of metal-organic frameworks (MOFs) so far has largely relied on nonspecific binding interactions to host small molecular guests. Here, long ( $c a .2$ nanometers) organic struts incorporating 34- and 36-membered macrocyclic polyethers as recognition modules were used in the construction of several crystalline primitive cubic frameworks that behave in a manner beyond open reticulated geometries. MOF-1001 is capable of docking paraquat dication $\left(\mathrm{PQT}^{2+}\right)$ guests within the macrocycles in a stereoelectronically controlled fashion. This act of specific complexation yields quantitatively the corresponding MOF-1001 pseudorotaxanes, as confirmed by X-ray diffraction and solidstate/solution nuclear magnetic resonance spectroscopic studies performed on MOF-1001, its pseudorotaxanes, and their molecular strut precursors. A control experiment involving the attempted inclusion of $\mathrm{PQT}^{2+}$ inside a framework (MOF-177) devoid of polyether struts showed negligible uptake of $\mathrm{PQT}^{2+}$, indicating the vital importance of the macrocyclic polyether in $\mathrm{PQT}^{2+}$ docking. 
The concept of architectural domains that operate independently, yet are interconnected, is common in biology but difficult to achieve in synthetic materials. We believe that 'concept transfer' from biology is a useful strategy toward achieving materials with higher complexity. Recently, we focused our attention on the design and synthesis of porous crystals composed of several architectural domains, one of which is capable of docking molecules in a manner akin to the well-known docking of drug molecules.

Our design takes advantage of the emerging chemistry of metal-organic frameworks (MOFs) (1-3), which has been used effectively to assemble components with simple constitutions - specifically, organic struts and inorganic joints into threedimensionally ordered structures. The vast majority of porous MOFs prepared thus far can be regarded (Fig. 1) as having two important architectural domains: the pore aperture which is responsible for the shape- and size-selective binding of incoming molecules (sorting domain) (2), and the internal surface of the pores onto which gases or small molecules can be compacted and distributed with simple interaction sites covering the struts and joints, and in some cases, the interaction is with open metal sites (coverage domain) (4-8). The synthesis of more complex MOFs, however, where more than two domains are present, remains unexplored. Here, we show how molecular recognition components, much used in supramolecular chemistry $(9,10)$, can be integrated in a modular fashion into struts of MOFs, thereby creating recognition sites into which incoming guests will dock in a highly specific manner with stereoelectronic control (active domain). In essence, the third domain combines shape, size and electronic 
elements in the recognition of incoming guests, and brings order to otherwise highly disordered guests in conventional MOFs, and thus this chemistry presents a class of MOFs that are beyond open reticulated geometries.

Specifically, we used the primitive cubic topology of the archetypical MOF-5 (11), in which benzene struts are joined by $\mathrm{Zn}_{4} \mathrm{O}\left(\mathrm{CO}_{2}\right)_{6}$ cluster joints, as the target for our design. Initially, we demonstrated the feasibility of using the long 1/4DMBDA (1) to make MOF-1000 (12), which has the MOF-5 topology, albeit quadruply interpenetrated (Fig. 2A). This approach was extended to the more complex struts, BPP34C10DA (2) and 1/5DNPPP36C10DA (3), which are known to act as electron-rich receptors for electrondeficient substrates (13), to make the corresponding MOF-1001A, MOF-1001 and MOF1002 (Fig. 2, B to D). We further show that indeed each of the crown ether receptors in MOF-1001 is accessible as evidenced by the docking of the paraquat dication $\left(\mathrm{PQT}^{2+}\right)$ at every one of the receptor sites. In contrast to known MOFs where the frameworks are used mainly as passive platforms for the adsorption of gases and molecules, MOF-1001 not only has active components in precise recognition sites but also, by virtue of the openness of its structure, allows substrates to diffuse freely from solution, through the pores, and finally dock at these active domains.

Crystals of MOF-1000 (14) (Fig. 2A and 3A) were obtained by mixing a solution of strut 1 (15), with $\mathrm{Zn}\left(\mathrm{NO}_{3}\right)_{2} \cdot 4 \mathrm{H}_{2} \mathrm{O}$, in $N, N$-diethylformamide (DEF) using conditions previously employed in the synthesis of MOF-5 $(5,11)$. Its crystal structure displays the same structural topology as does MOF-5. It is found to be four-fold interpenetrated because of the length and slender nature of the strut - the distance between the two 
carbon atoms from the carboxylate groups is $19.3 \AA$. Successful crystallization of MOF1000, however, confirmed for us the practicality of creating MOFs with higher complexity using this synthetic protocol.

The struts $\mathbf{2}$ and $\mathbf{3}$, containing 34- and 36-membered polyether rings, which have been extensively used (13) as receptors for the wide range of electron-deficient substrates, are ideally suited as molecular recognition modules for making MOFs. Strut 2 was prepared by means of a convergent synthetic approach (14), and used under similar conditions to those employed in the synthesis of MOF-1000 to give MOF-1001A and MOF-1001 (14). The crystal structure of MOF-1001A is a triply interpenetrating framework (Fig. 2B and 3B), whereas that of MOF-1001 is the corresponding noninterpenetrating form (Fig. 2C and 3C); both have the MOF-5 type topology. The existence of MOF-1001A, despite its occasional appearance as a minor product, validates indirectly the high porosity of MOF-1001. The sheer openness of the structure, however, led us to optimize the reaction conditions yet further in order to successfully obtain MOF1001 as a pure phase (14). MOF-1001 has $F m \overline{3} m$ symmetry, with an exceptionally large unit cell parameter $a=52.93 \AA$. Furthermore, we extended the methodology to the synthesis of MOF-1002 (Fig. 2D and 3D) by using the 1,5-dioxynaphthalene-containing strut 3, which was produced via a divergent synthetic route (14). Single crystal X-ray diffraction studies (14) indicate that MOF-1002 shares an identical cubic backbone with MOF-1001, affirming the generality of such a methodology for building a variety of crystalline structures with such long struts capable of molecular recognition. 
Calculations of the volumes of open space within the MOF structures confirm the highly open nature of these crystals (86.9\% space unoccupied by MOF-1001 framework atoms, based on a model using the program Cerius ${ }^{2}$, version 4.2). The inherent flexibility of the macrocyclic polyether substructure was evident from the single crystal X-ray analysis of MOF-1001. The bismethylenedioxy units of the tetraethylene glycol loops in the substructure are found to be highly disordered. Nevertheless, the positions of all the atoms in the inorganic joints and the rigid backbone of the links are unambiguous as judged by comparison of the resulting bond distances and angles with the model structure (16). Based on the overall geometry and stoichiometry of the MOF framework, we can conclude that the crown ether receptors - capable of the complexation behavior required (17) for molecular recognition — are integrated precisely and periodically inside a robust framework. Thus, the extended framework provides the basis for their strategic placement so that they are exposed to the maximum accessibility to guests in threedimensional space.

To date, a number of reports (18-20) have appeared on the synthesis and structure of hybrid organic-inorganic compounds with macrocycles and mechanically interlocking components. The MOFs presented here combine the precise positioning of the active domains with docking as an expression of molecular recognition. This property was revealed by examining the molecular recognition behavior of the macrocyclic polyethers 2 and 3 as docking sites. When MOF-1001 crystals were introduced into a saturated solution of $\mathrm{PQT} \cdot 2 \mathrm{PF}_{6}$ in acetone, the crystals immediately turned red, and the color intensified over sixty minutes (21) (Fig. 4, A to E, and Movie S1) - a typical behavior 
for this binding event that indicates significant charge-transfer interactions (22) between $\mathrm{PQT}^{2+}$ and crown ether rings. This observation points to the formation of MOF-1001 pseudorotaxanes (23) by threading of $\mathrm{PQT}^{2+}$ through the middle of the crown ether. The reversibility of such process was evidenced by the reappearance of the original light yellow color upon rinsing with acetone, where sixty percent of $\mathrm{PQT}^{2+}$ could be removed after rinsing MOF-1001 pseudorotaxanes $(2.8 \mathrm{mg}$ ) four times with one milliliter every thirty minutes (14). The complexed MOF-1001 maintained the original high crystallinity of the parent framework, as confirmed by coincident powder X-ray diffraction (PXRD) patterns.

Further evidence of complexation was obtained by examining the ${ }^{1} \mathrm{H}$ NMR spectrum of the MOF-1001 pseudorotaxanes after dissolution in $\mathrm{DCl}$ (14). Integration of the peaks appearing at $7.96 \mathrm{ppm}\left(\mathrm{d}, 4 \mathrm{H}, \mathrm{Ar}-\mathbf{H}^{\mathrm{a}}\right.$ in $2,{ }^{3} J=8.5 \mathrm{~Hz}$, see Fig. $\left.4 \mathrm{~F}\right)$ and 4.60 ppm (s, 6H, N-CH $\mathrm{H}_{3}$ in $\mathrm{PQT}^{2+}$ ) revealed the expected 1:1 ratio of strut 2 and $\mathrm{PQT}^{2+}$, indicating that the docking phenomenon of $\mathrm{PQT}^{2+}$ does indeed take place at every crown ether ring throughout the whole MOF framework (fig. S10). Solid-state ${ }^{15} \mathrm{~N}$ NMR spectroscopy, a technique which is highly sensitive to the environment of the nitrogen $\left({ }^{15} \mathrm{~N}\right)$ in $\mathrm{PQT}^{2+}$, provided further strong evidence for docking in MOF-1001. Isotopelabeled $\mathrm{PQT}^{2+}$ (14) with $25 \%$ abundance of ${ }^{15} \mathrm{~N}$ was used to make the MOF-1001 pseudorotaxanes, and the resulting solid was examined by ${ }^{15} \mathrm{~N}$ cross-polarization magicangle spinning (CP/MAS) spectroscopy (24). The spectrum of the uncomplexed $\mathrm{PQT}^{2+}$ has a ${ }^{15} \mathrm{~N}$ signal centered on $207.2 \mathrm{ppm}$, whereas the spectrum of $\mathrm{PQT}^{2+}$, bound within the crown ether rings in MOF-1001, shows a significant upfield shift to $204.6 \mathrm{ppm}$ for the 
${ }^{15} \mathrm{~N}$ resonance resulting from docking into the macrocyclic polyether units of the struts (Fig. 4G)

Similar studies carried out on strut $\mathbf{2}$ were used as a molecular analogue for comparison with MOF-1001 complexation experiments. Here, addition of $\mathrm{PQT} \cdot 2 \mathrm{PF}_{6}$ to an acetone solution of strut 2 led to the formation of a pseudorotaxane $[\mathrm{PQT} \subset 2] \cdot 2 \mathrm{PF}_{6}$. The binding affinity $\left(K_{\mathrm{a}}=829 \mathrm{M}^{-1}\right)$ (fig. S2) between $\mathrm{PQT}^{2+}$ and strut $\mathbf{2}$ in solution was obtained from spectrophotometric titrations. Single crystal X-ray diffraction of the $[\mathrm{PQT} \subset 2] \cdot 2 \mathrm{PF}_{6}$ (Fig. 4H) clearly shows the insertion of the $\pi$-electron deficient bipyridinium dication through the middle of the macrocyclic polyether. $\pi-\pi$ stacking and $[\mathrm{C}-\mathrm{H} \cdots \mathrm{O}]$ interactions are reflected in the interplanar separation of $3.6 \AA$ between the bipyridinium unit of $\mathrm{PQT}^{2+}$ and the hydroquinone rings. The same upfield shift trend in the ${ }^{15} \mathrm{~N}$ NMR spectra observed for MOF-1001 pseudorotaxanes was also evident in the ${ }^{15} \mathrm{~N}$ NMR spectra of $[\mathrm{PQT} \subset 2] \cdot 2 \mathrm{PF}_{6}$ in the solid state (14) (Fig. 4I) as well as in solution (fig. S5). Control experiments were carried out by attempting to introduce PQT $2 \mathrm{PF}_{6}$ into porous MOF-177 crystals (25), the pore dimensions ( $d=11.8 \AA$ ) of which were expected to allow the free movement of $\mathrm{PQT}^{2+}$ within the pores. In the event, we established that less than $0.06 \mathrm{PQT}^{2+}$ per strut of MOF-177 were incorporated in the pores (fig. S11). These results show clearly that specific stereoelectronic host-guest interactions, rather than just simple diffusion and adsorption, are responsible for the all but quantitative formation of the MOF-1001 pseudorotaxanes. 
1. O. M. Yaghi et al., Nature 423, 705 (2003).

2. S. Kitagawa, R. Kitaura, S. Noro, Angew. Chem., Int. Ed. 43, 2334 (2004).

3. G. Férey et al., Science 309, 2040 (2005).

4. A. G. Wong-Foy, A. J. Matzger, O. M. Yaghi, J. Am. Chem. Soc. 128, 3494 (2006).

5. M. Eddaoudi et al., Science 295, 469 (2002).

6. Z. Wang, S. M. Cohen, Chem. Soc. Rev. 38, 1315 (2009).

7. J. Lee et al., Chem. Soc. Rev. 38, 1450 (2009).

8. J. Rowsell, E. Spenser, J. Eckert, J. A. K. Howard, O. M. Yaghi, Science 309, 1350 (2005).

9. J. M. Lehn, Science 227, 849 (1985).

10. J. F. Stoddart, Nature Chem. 1, 14 (2009).

11. H. Li, M. Eddaoudi, M. O’Keeffe, O. M. Yaghi, Nature 402, 276 (1999).

12. We assign MOF-1000 numbering scheme to MOFs that are constructed from struts capable of stereoelectronically controlled binding.

13. J. F. Stoddart, D. B. Amabilino, Chem. Rev. 95, 2725 (1995).

14. Materials and methods, including full description of the synthesis and characterization, are available as supporting material on Science Online.

15. Strut 1 was synthesized (14) by the attachment of 4-(carboxyphenyl)ethynyl groups to the 2- and 5-positions of a central 1,4-dimethoxybenzene ring using the Pd-catalyzed alkyne-aromatic Sonogashira coupling (26).

16. It has not escaped our attention that the phenylene rings that incorporate the struts in 2 and 3 also support planes of chirality. As a consequence, there is yet another 
fundamental source of disorder associated with the polyether loops in MOF-1001A, MOF-1001, and MOF-1002. Moreover, there is also the prospect of being able to construct chiral MOFs where the elements of chirality are planar (27) in origin.

17. V. Balzani, A. Credi, F. M. Raymo, J. F. Stoddart, Angew. Chem. Int. Ed. 39, 3348 (2000).

18. S. J. Loeb, Chem. Commun. 1511 (2005).

19. K. Kim, Chem. Soc. Rev. 31, 96 (2002).

20. C.-F. Lee et al., Nature 458, 314 (2009).

21. Crystals of MOF-1001 were first immersed in acetone to exchange the $N, N$ dimethylformamide (DMF) guests and unreacted BPP34C10DA. This process was repeated nine times by decanting and refreshing with acetone $(5 \mathrm{~mL})$ every thirty minutes to ensure full exchange of DMF.

22. D. B. Amabilino et al., J. Am. Chem. Soc. 117, 11142 (1995).

23. P. R. Ashton, D. Philp, N. Spencer, J. F. Stoddart, J. Chem. Soc., Chem. Commun. 23, 1677 (1991).

24. The experiments were done by introducing acetone-exchanged MOF-1001 into acetone solutions of PQT $2 \mathrm{PF}_{6}$ with different amounts of $\mathrm{PQT}^{2+}$. After sitting for $6 \mathrm{~h}$, solvent was then removed by evaporation and the residue was further dried under vacuum $\left(10^{-2}\right.$ Torr $)$ overnight at room temperature. An ${ }^{15} \mathrm{~N}$ CP/MAS NMR spectrum was acquired on the solid sample and the loading of $\mathrm{PQT}^{2+}$ was determined by solution-state ${ }^{1} \mathrm{H}$ NMR spectroscopy after digestion of the solid. See supporting online material Section S3 for information. 
25. H. Chae et al., Nature 427, 523 (2004).

26. K. Sonogashira, Y. Tohda, N. Hagihara, Tetrahedron Lett. 50, 4467 (1975).

27. V. Prelog, G. Helmchen, Angew. Chem. Int. Ed. 21, 567 (1982).

28. The work was supported by U.S. Department of Defense (DTRA: HDTRA1-0810023) and Northwestern University. We thank Ms. Sanaz Kabehie for assistance with emission spectroscopy. Crystallographic data for $[\mathrm{PQT} \subset 2] \cdot 2 \mathrm{PF}_{6}, \mathrm{MOF}-1000$, MOF1001A, MOF-1001, and MOF-1002 have been deposited into the Cambridge Crystallographic Data Centre (CCDC) under deposition numbers CCDC 728413 to 728420. 


\section{Supporting Online Material}

www.sciencemag.org

Materials and Methods

Figs. S1 to S24

Tables $\mathrm{S} 1$ to $\mathrm{S} 11$

Schemes S1 to S13

Movie S1

References 


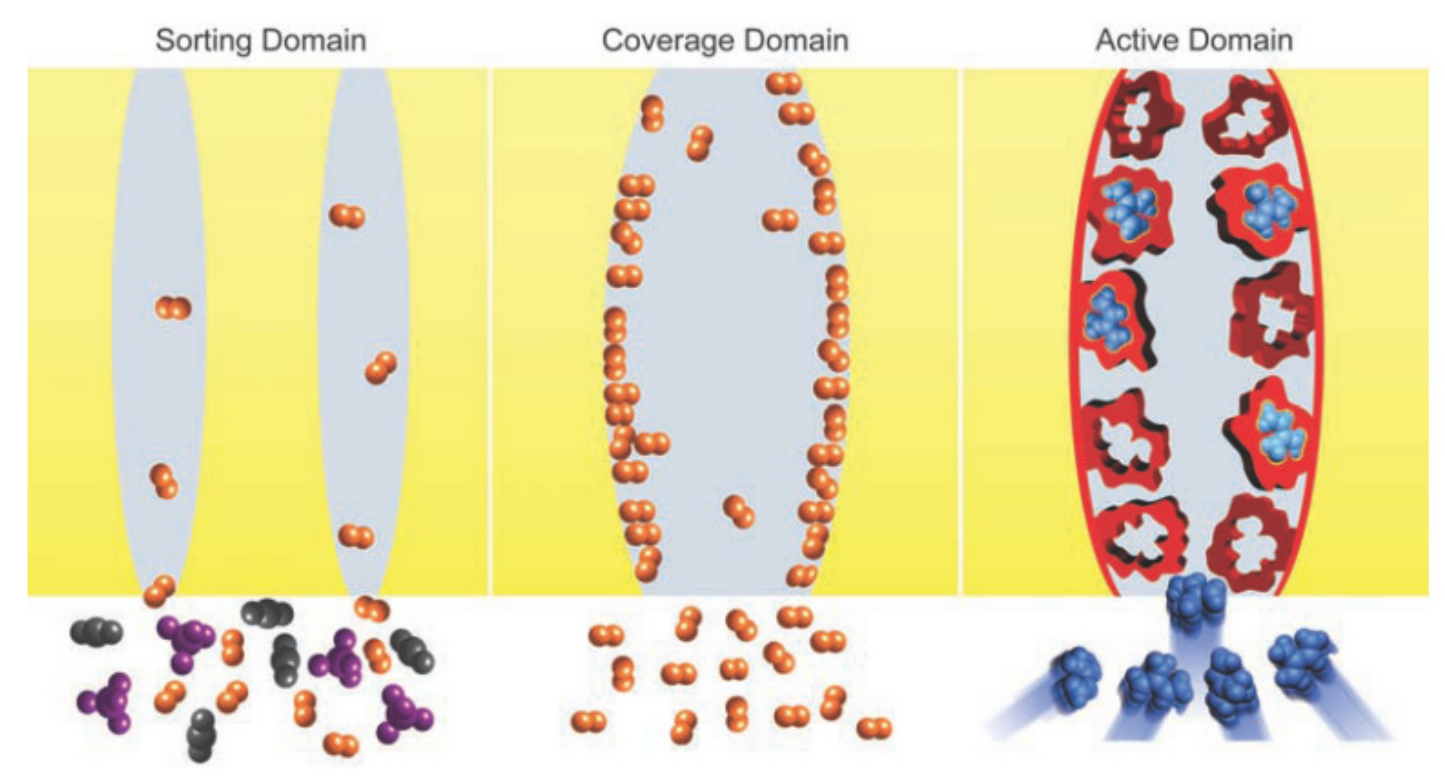

Fig. 1. Classification of the different porous domains in metal-organic frameworks. In the Sorting Domain, guest molecules are selected according to their size at the orifices of the pores. The entry of $\mathrm{H}_{2}$ (orange) and concomitant exclusion of $\mathrm{CO}_{2}$ (black) and $\mathrm{CH}_{4}$ (purple) reflects the sieving-like action at the entrances to the pores. In the Coverage Domain, the guest molecules along the walls of the pores are disordered on account of their weak non-specific interactions with the framework surrounding the pores. By contrast, the Active Domain has built-in recognition sites which help to maneuver and dock incoming guests in a highly selective and stereoelectronically controlled manner. These recognition sites (red) could be $\pi$-electron rich and, as such, would seek out $\pi$ electron deficient substrates (blue). 
A
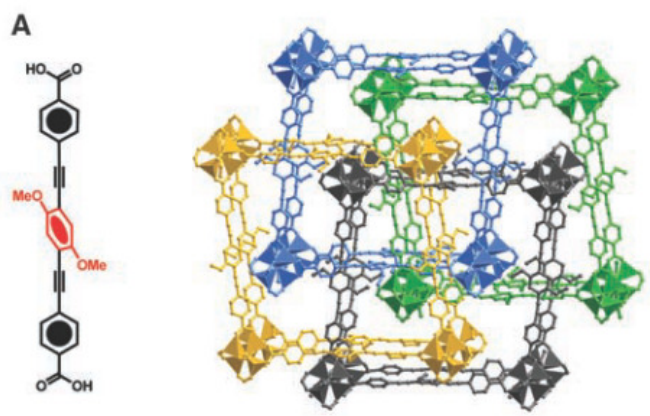

MOF-1000

1/4DMBDA (1)

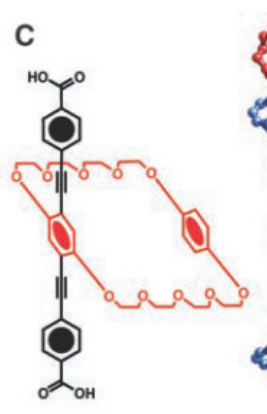

BPP34C10DA (2)

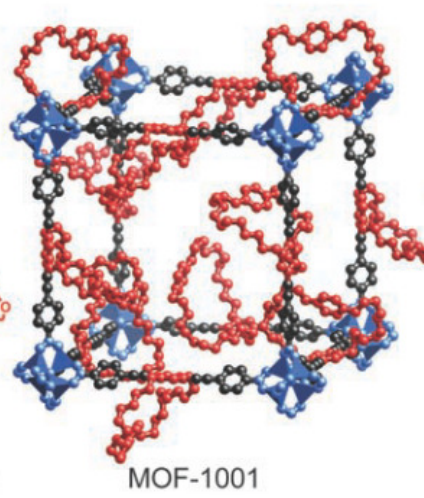

B

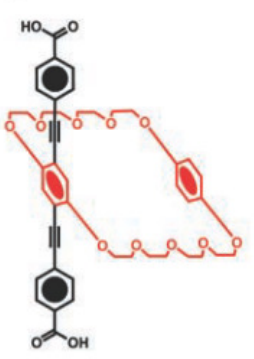

BPP34C10DA (2)

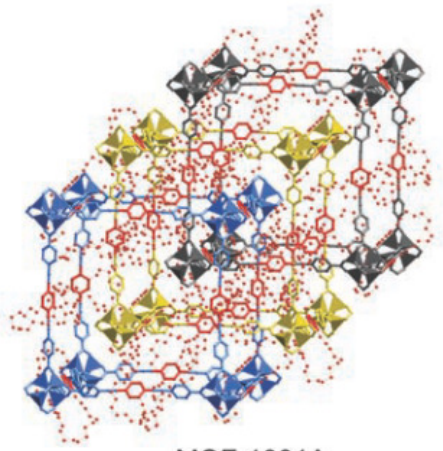

MOF-1001A

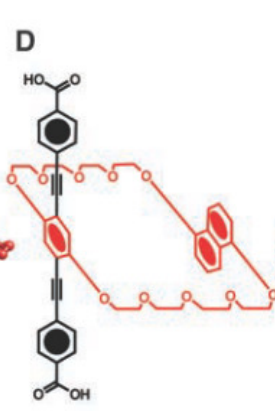

1/5DNPPP36C10DA (3)

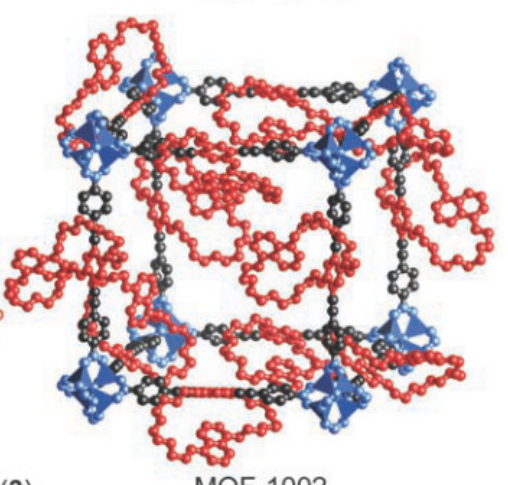

MOF-1002

Fig. 2. Ball-and-stick drawings of single crystal structures of MOF-1000, MOF-1001A, MOF-1001, MOF-1002, and their corresponding organic struts. Strut 1 was employed to obtain MOF-1000 (A), which has a four-fold interpenetrating structure with different frameworks shown in four different colors. The crystal structure of MOF-1001A from strut $2(\mathbf{B})$ is a triply interpenetrating cubic structure (shown in blue, gold and gray), with polyethers represented by red balls and wires. MOF-1001 from strut $2(\mathbf{C})$, and MOF1002 from strut 3 (D) share the identical cubic framework backbone, and crown ethers are placed precisely throughout the whole framework $\left(\mathrm{Zn}_{4} \mathrm{O}\left(\mathrm{CO}_{2}\right)_{6}\right.$ polyhedra, blue; organic struts, gray; crown ethers, red). Crown ethers in all the structures were modeled by Cerius ${ }^{2}$. All hydrogen atoms have been omitted for clarity. 
A

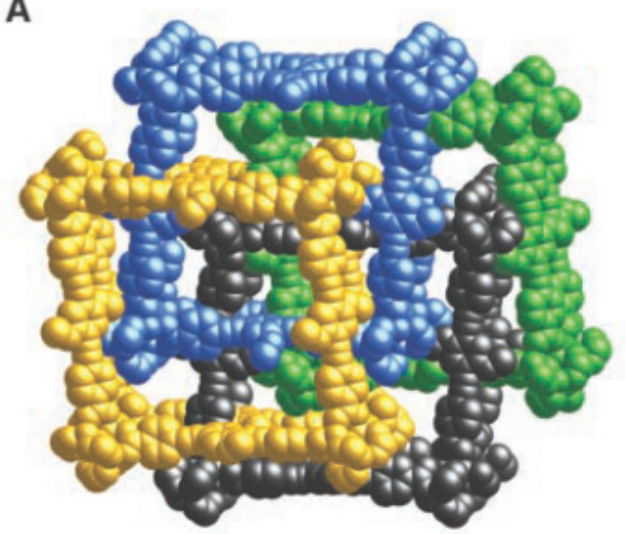

C

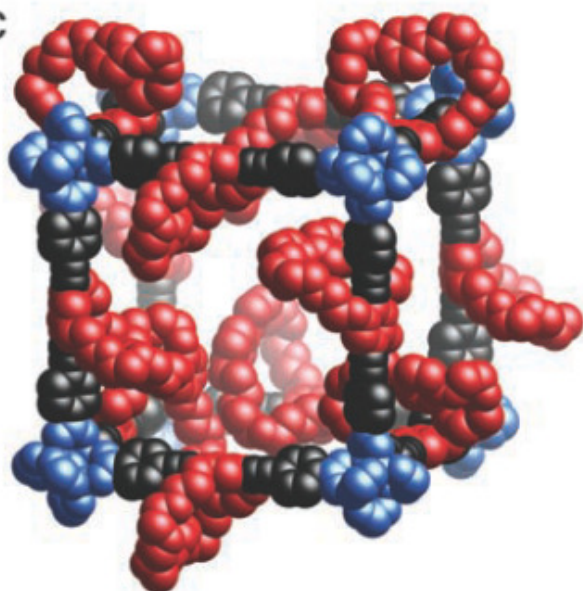

B
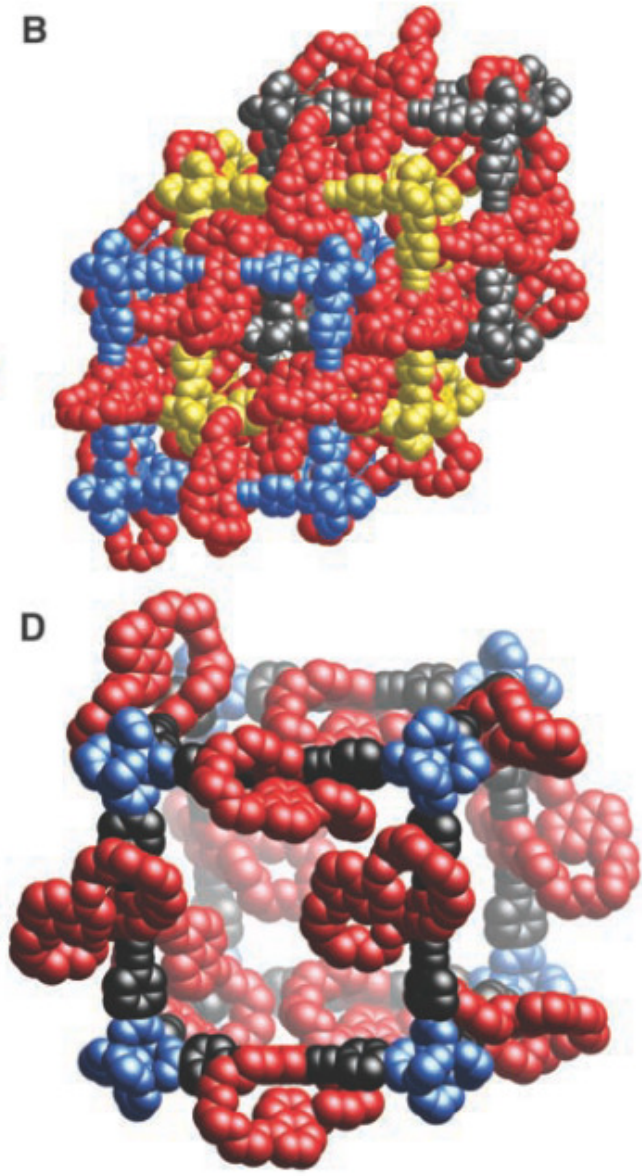

Fig. 3. Space filling illustration of MOF-1000, MOF-1001A, MOF-1001, and MOF-1002. The length of the struts $(19.3 \AA)$ allows the structures of MOF-1000 (A) and MOF1001A (B) to interpenetrate. In contrast, high volumes of open space were present in the non-interpenetrating MOF-1001 (C) and MOF-1002 (D) synthesized from struts with the same length. This feature ensures the full accessibility of the electron-donating receptors for the incoming substrates within the pores. The same color codes as in Fig. 2 were applied. All hydrogen atoms have been omitted for clarity. 

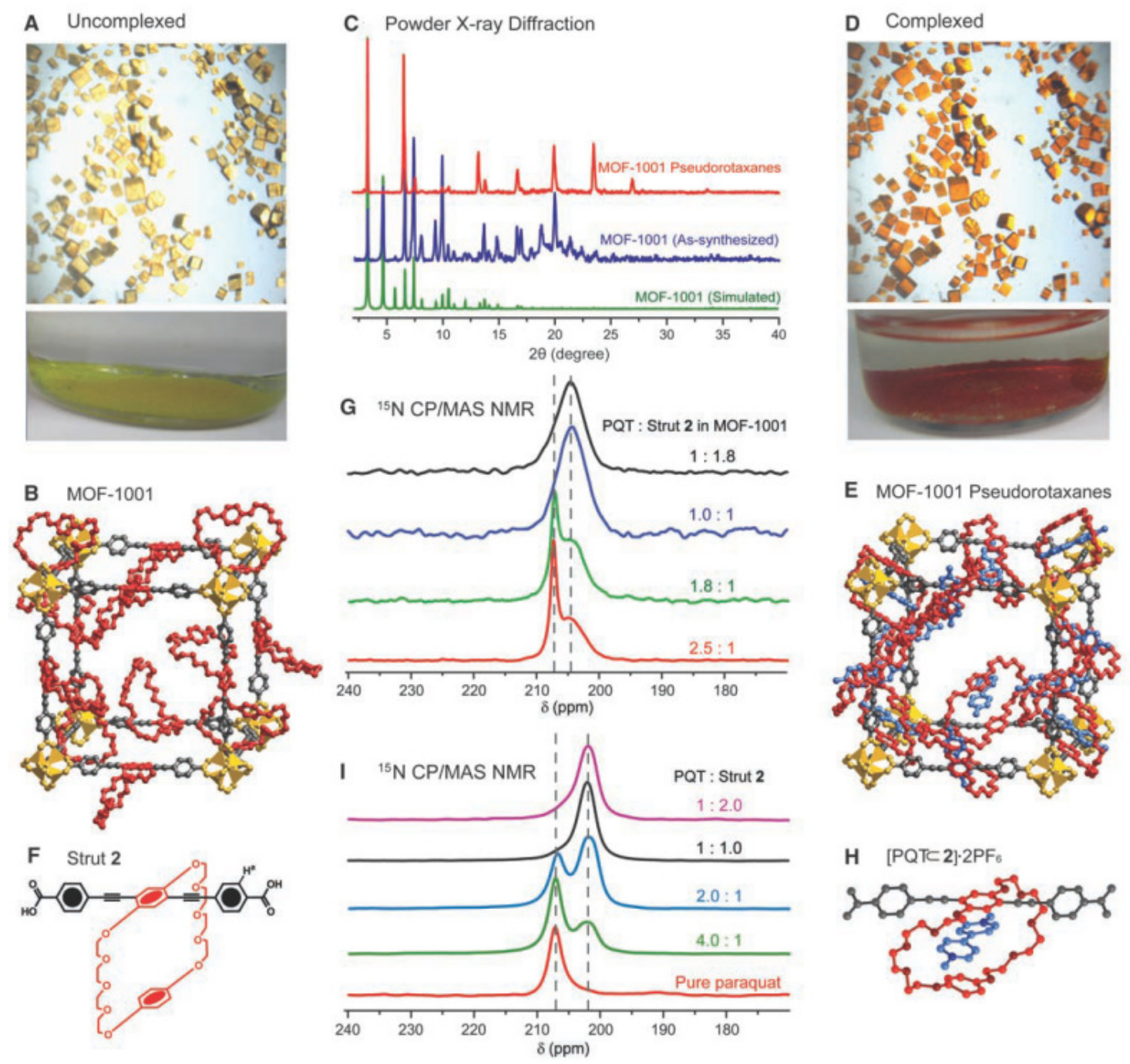

Fig. 4. X-ray diffraction and solid-state NMR spectroscopic studies on MOF-1001, MOF1001 pseudorotaxanes, and their molecular analogues. MOF-1001 (A, B) maintained its crystallinity after docking of $\mathrm{PQT}^{2+}$, a single-crystal-to-single-crystal transformation revealed by the X-ray diffraction pattern $(\mathbf{C})$. The crystal structure of MOF-1001 and the simulated MOF-1001 pseudorotaxanes structure $(\mathbf{D}, \mathbf{E})$ are illustrated in ball-and-stick models. This quantitative threading was confirmed by the $1: 1$ stoichiometry of $\mathrm{PQT}^{2+}$ and strut $2(\mathbf{F})$. This docking phenomenon resulted in the upfield shifts of the ${ }^{15} \mathrm{~N}$ CP/MAS 
signals $(\mathbf{G})$ (11) compared to the free $\mathrm{PQT}^{2+}$. The molecular pseudorotaxane analogue $[\mathrm{PQT} \subset 2] \cdot 2 \mathrm{PF}_{6}(\mathbf{H})$ was found to have the same upfield shift trend $(\mathbf{I})(\Delta \delta=4.9 \mathrm{ppm})$. Color schemes: $\mathrm{Zn}_{4} \mathrm{O}\left(\mathrm{CO}_{2}\right)_{6}$ polyhedra, gold; organic struts, gray; crown ethers, red; $\mathrm{PQT}^{2+}$, blue. All hydrogen atoms and counter ions have been omitted for clarity. 